\title{
Guiding Functions of Basic Physical Ideas on Research and Innovation
}

\author{
Chunqing $\mathrm{Xu}$ \\ School of Physics, University of Electronic and Science \\ Technology of China \\ Chengdu, China \\ E-mail: Lihx176@163.com
}

\begin{abstract}
The thoughts decide the behaviors, so basic physical ideas are footholds for us to be engaged in scientific research, and they are also the keys to improve problem-analyzing skill and develop creative thinking and become an innovative talent. This article discussed the guiding functions of basic physical ideas on research and innovation from four aspects: the source of the theory, the test of the theory, the development of the theory and the evaluation of the theory.
\end{abstract}

Key word-- basic ideas; laws; theorem; physics

\section{INTRODUCTION}

Physics explores the basic laws in the objective world. It is the bases of other natural sciences and all engineering technologies. It is a required course for the students of science and engineering specialities. However, some very important basic ideas implied in the physical theories are also very important. The thoughts decide the behaviors, so it goes without saying that these basic ideas are of guiding functions on research and innovation. Today, science and technology have developed considerably. The demand for innovative talents is more and more urgent, and the cultivation of innovative talents also becomes a common concern topic to masses of educators. The study on the relationship between the basic physical ideas and innovation talents is seldom involved, and there is no full revelation for their close link although theorists have made a lot of discussion on them(for example: Song 2002; Zhou 2002; Zhao 2005; Zhu 1999; Wang 2006). This article discussed the guiding functions of basic physical ideas on research and innovation from four aspects: the source of the theory, the test of the theory, the development of the theory and the evaluation of the theory.

\section{BASIC PHYSICAL IDEAS}

\section{A. The Source of the Theory}

Physics composed by physics laws (or principles) and theorems has developed into a systematic science with strict logic. The laws are summed up from the practice. The theorems are strictly deduced by the laws. Therefore, it is obvious that the ultimate source of physical theory is the practice. In physics, if the theory system can be likened to a big tree, the laws are its roots, the theorems derived from the laws are just like its trunks and branches, and the practical activities of people are its survival soil. If the root of the theory system and its trunks and branches could not be distinguished during learning, it is naturally difficult to understand it and use it well. Thus, an important way of systematizing knowledge is to distinguish the roots and branches of the theory system.

\author{
Hongchun Yang \\ School of Physics, University of Electronic and Science \\ Technology of China \\ Chengdu, China \\ E-mail:519634262@qq.com
}

It should be noted that some theorems are called laws or principles, while some principles or laws are called theorems due to history and habits and other reasons. Therefore, the roots and the branches of theory system should be distinguished by their sources and tests, not by their names alone.

Case 1: Why is the acceleration of an object proportional to the external force but inversely proportional to the mass of the object? Why can a changing magnetic field generate a vortex electric field in the space? These problems are the mistakes many people often make. This is led by an unclear understanding on the sources of theory. The Laws is summed up from practice and is not derived, so there is no "why."

\section{B. The Test of the Theory}

Since the theorems is deduced by the laws, whether it is correct or not depends on whether the derivation process and the laws are correct or not, and whether the laws is correct or not is judged by the practice, so the final standard of testing theory is the practice. The theory comes from practice and must be constantly tested by the practice, and this is the sole criterion for testing the theory.

People often call a law a hypothesis when advancing it, and it is called law or principle if it passes practice examination. For example, the special theory of relativity is built on two assumptions. Nowadays, the special theory of relativity has been proved by a large number of facts and is generally accepted by people, and the two basic assumptions have been known as the principle of the constancy of light velocity and the relativity principle. It must be pointed out that people generally use the relativity principle to test a new theory. This approach is reasonable, but the ultimate inspection standard of a new theory is the practice. Evidently, we cannot consider that a new theory is untenable if it conflicts with the relativity principle, but can withstand the test of the practice.

The above ideas about the source and test of the theory are the basic ideas formed in people's long-term practice. A lot of people do not understand these ideas, so they take detours and wrong paths in actual research work. It is very easy to encounter this kind of examples, such as trying to prove basic principles; the basic assumptions of theory is not derived from practice, but from subjective understanding; doubting and criticizing others' theories is not based on the practice, but on speculativeness alone.

Case 2: Are some principles (or laws), the academic authorities and other concepts that people are convinced of for a long time absolutely reliable? The answer is negative, because the practice is the sole criterion for testing the theory. An innovative talent must possess this basic idea, because 
innovation needs to have a free hand, and revering the tradition theory and the authority inevitably shackle thinking.

\section{The Development of the Theory}

Human's practical activities are subject to the limitations of material conditions and technical means at a certain periods, so people's practical activities are gradually deepened with the continuous improvement of material conditions and technical means. This causes that the phenomenon inconsistent with the original laws may appear as new phenomenon and new experiment are discovered continually. People need to amend these laws or advance a new hypothesis, and the new hypothesis will become a new law if withstanding the testing of practice. Therefore, the development of the theory has become a process of gradually approaching objective truth. It is just as Popper's point of view: "any theory is not absolutely eternal truth but only a temporary assumption" (Ge 1994).

There must be a connection between a new theory and an original theory, that is, the new theory will indicate that the original theory is applicable to some range. For instance, the special theory of relativity points out that Newton's law applies to low-speed situations. Without this connection, a new theory cannot explain the fact the original theory can explain, thereby, a conflict will be engendered between the new theory and the practice.

Over a century ago, a variety of physical phenomena people found could all get perfect explanations. A lot of people thought that the physics mansion had been established, and there was not much valuable work left. However, people found a series of phenomenons that could not be explained by using physics at that time, such as photoelectric effect and atomic structure models. These new phenomena just led to the birth of quantum mechanics and the special theory of relativity, and people realized Newton's laws are only the laws that macroscopic body approximatively complies with in the low-speed case, and people's understanding on the development of theory was further deepened again in turn. People recognize that quantum mechanics and the special theory of relativity are also the theories under certain conditions, and more perfect theories will also be born in the future.

It is hard to make important contribution in research if this basic idea of the physics theory cannot be understood well, because it is impossible to make a breakthrough on the original theory and create a new theory if the original theory is worshiped as "Bible".

Case 3: Will the special theory of relativity be abandoned if the velocity of matter may be superluminal in the future? The answer is negative, and this phenomenon will be an experimental basis for the birth of a new theory in the future. The new theory will point out that the special theory of relativity is the approximation theory in some range, and is still applicable in the range.

\section{The Evaluation of the Theory}

The sole criterion for testing a theory is the practice, but that's not all for the evaluation criteria of a theory. The evaluation criteria of a theory can be defined according to different perspectives and different disciplines respectively, but they should generally include the following three most general criteria: the practice basis, a wide range of applications and the simple logic with convenient application. The practice is the sole criterion for testing the theory, so the theory naturally cannot keep a foothold without the practice basis. The theory also has an issue about the range of application. The application range of Newton's laws is macroscopic objects in the low-speed case, so it is necessary to consider the relativistic effects in the high-speed case, and the quantum mechanics should be used in the micro field. It is very successful for the ideal gas model to interpret the gas pressure and temperature, but it is necessary to consider the molecule structure when interpreting the gas energy. Examples like this can be seen everywhere, and this also shows that right or wrong are not suitable to evaluate a theory, because it is wrong beyond the range of application. Of course, it is still temporarily unclear what the application range of some theories is, but people will gradually know them with the development of the science. In fact, countless scholars have proposed a variety of theories in the long history process of the scientific development, and those with wide application range, small imperfections, simple logic and convenient application are often widely used. Contrary, the theories without these features are still shelved, even though they are able to explain some facts.

Case 4: Is a profound theory using very complex math a great theory? The answer is negative, because the theory is not evaluated by whether it uses very complex mathematical tool or whether it is inscrutable. The theories with simple logic and convenient applications are worthy of our favour.

This basic idea shows that we should not be overly demanding on a theory when evaluating it though we should strive to make a theory perfect when putting forward it. If we are deliberately mystifying, make a new problem intricate and completely negate a new theory when it has some problems regardless of its merits, these behaviors are not desirable, and an innovative talent should not act like this.

The above physical ideas were synthesized to analyze a practical example. Chinese scholar named Tan Shusheng founded the "standard space-time theory", and think that this theory is better than the special theory of relativity in logical simplicity and satisfactorily explains all previous experimental results and foresees some facts that are not predicted so far. The theory is built on four basic assumptions: (1) There is a standard inertial reference system in all inertial systems, and the one-way light speed is isotropic in this reference system; (2) The average velocity of light along any closed loop is $\mathrm{C}$ in vacuum in any inertial reference system, and it has nothing to do with the motion state and spatial orientation of the light source; (3) There is no a upper limit for the velocity of matter in any inertial reference systems; (4) The new space-time transformation at low speeds must be reverted to Galilean's transformation.

Case 5: Is there anything wrong with the "standard space-time theory" only from the view of basic assumptions? Regardless of the first two assumptions, the third hypothesis negates the principle of constancy of light velocity. This assumption is not based on the practice because the superluminal matter is still not found by the academic community. The theory comes from the practice, so the theory without a practice basis naturally can not keep a foothold. The fourth assumption is not necessary because there must be a connection between a new theory and an original theory. The new space-time transformation must violate the practice if it cannot be reverted to Galilean's transformation at low speeds. According to this case, it is not difficult to realize how important the above basic physical ideas are for the people to research and innovate. 


\section{CONCLUSIONS}

To sum up, the theory comes from the practice, and the practice is the sole criterion for testing the theory. The development of the theory is a process of gradually approaching objective truth. The general evaluation criteria of the theory are the practice basis, the wide range of applications and the simple logic with convenient application. These basic physical ideas are not only applied to physics but also to other natural sciences. It is very important to master these basic ideas, thus, we no longer just passively accept the knowledge, and initiative in learning can be stimulated so that we are of a deeper understanding on the theories. These basic ideas help to emancipate our minds, cultivate creative thinking and avoid the impingement and the detour in research. In short, the thoughts decide the behaviors. These ideas about the physical theory are the basic ideas that we should possess when learning scientific knowledge. They are the foothold when engaging in scientific research and the qualities the creative talents of the new century must possess.

\section{REFERENCE}

[1] Song, Z. H, "The rise of research on complexity and the choiceless of college physical view," 3rd ed, The impact of science upon society, 2000, pp.13-16.

[2] Zhou, J. S, "The view of nature in the book of changes and the Philosophical basis in modern physics," 2rd ed, Journal of Shanxi Teachers' College, 2002, pp.6-10.

[3] Zhao, Z, "Einstein and the breakthrough in the physical view," 12rd ed, College physics, 2005, pp.5-11.

[4] Zhu, H. L, and Cheng, X. Y, "The innovative spirit of physics," 12rd ed, College physics, 1999, pp.38-41.

[5] Wang, J. Y, and Zhang, X. Y, "Depend on Key Subjects, interact with teaching and research, cultivate innovative talents," $11 \mathrm{rd}$ ed, College physics, 2006, pp.59-66.

[6] Ge, P., and Chen, X. X. (1994). The review of Popper's Science development mode. Journal of Anhui Agricultural University, 1, 43-47. Tan, S. S. (1984). Standard space-time theory. Journal of National University of Defense Technology, 1, 1-13 